(J. Ped. Ortho. J Pediatr Orthop 2007; 27:898). In addition, femoral head DWI was used to calculate apparent diffusion coefficient (ADC). For each measurement the left and right were compared using within subject coefficient of variation (WS CV).

Results 44 neonates were imaged. Median (IOR) gestational age at birth was 36 weeks (range 25-42 weeks), 13 less than 32 weeks gestation; median gestational age at scanning was 41 weeks (range 37-46). 18 babies had HIE of whom 13 were cooled. Mean (SD) and WS CV $(95 \% \mathrm{CI})$ for the following measurements were: Femoral head ADC 1.87 (0.094) on Right, 1.87 (0.099) on Left, WS CV 2.42 (2.12-2.72); acetabular width 17.1 (1.6) on Right, 17.0 (1.6) on Left, WS CV 3.50 (3.02-3.96); acetabular depth 3.81 (0.81) on Right, 3.81 (0.81) on Left, WS CV11.6 (10.1-13.1); acetabular width: depth ratio 4.66 (1.0) on Right, 4.66 (1.1) on Left, WS CV 13.6 (11.9-15.2).

Conclusions In this diverse group of babies the variation between sides was minimal. Analysing a single hip should be sufficient in this population.

\section{COMPARISON OF INTENSIVE LIGHT-EMITTING DIODE AND INTENSIVE COMPACT FLUORESCENT PHOTOTHERAPY IN NON-HEMOLYTIC JAUNDICE}

doi:10.1136/archdischild-2012-302724.1326

S Takci, S Yigit, G Bayram, A Korkmaz, M Yurdakok. Hacettepe University Ihsan Dogramaci Childrens' Hospital, Ankara, Turkey

In severe and rapidly increasing jaundice, the use of high-intensity phototherapy provides greater effectiveness and a faster decrement in bilirubin levels. The aim of this study is compare the effectiveness of intensive compact fluorescent tube (CFT) and intensive LED phototherapy in higher doses of irradiance.

Method Forty three infants over 35 weeks of gestation with severe severe nonhemolytic hyperbilirubinaemia were enrolled in the prospective study. All infants received multidirectional (circular shaped) high-intensity phototherapy. Of these 20 infants received CFT while 23 infants received LED phototherapy. Bilirubin levels and body temperatures were measured periodically and the rates of bilirubin decrement were calculated.

Results Mean serum bilirubin level of the 43 infants was $20.5 \pm 1.5$ $\mathrm{mg} / \mathrm{dl}$ at the beginning of the therapy and mean duration of phototherapy was $20.6 \pm 1.1$ hours. The rate of mean bilirubin decline was $47.2 \%$ and the declination was more prominent in the first four hours. Clinical characteristics, initial bilirubin levels, rates of bilirubin decrement and the phototherapy durations were comparable for LED and CFT groups. Slightly elevated mean body temperature $\left(37.1^{\circ} \mathrm{C}\right)$ was determined in CFT group $(\mathrm{p}<0.05)$.

Conclusion Circumferential phototherapy units are effective devices, can provide up to $50 \%$ reduction in bilirubin levels within 24 hours in infants with nonhemolytic jaundice. Since it was shown that these devices can provide rapid decrease in bilirubin levels in the first few hours, they are useful in cases with high risk of bilirubin encephalopathy and kern icterus. These units decrease the hospitalization period so can help to maintain breast feeding.

\section{AUDIT ON MANAGEMENT OF NEONATAL JAUNDICE}

doi:10.1136/archdischild-2012-302724.1327

${ }^{1,2} \mathrm{R}$ Ravindran, ${ }^{2} \mathrm{~T}$ Ashraf. ${ }^{1} \mathrm{NICU}$, Homerton University Hospital NHS Trust; ${ }^{2}$ Paediatrics, Whipps Cross University Hospital NHS Trust, London, UK

Background Neonatal jaundice is a common medical problem in postnatal wards. With the introduction of NICE guidelines in UK, the management of jaundice of newborn infants has achieved clarity. Aims An audit was undertaken to check if local team were adhering to the NICE guidelines on neonatal jaundice management (May 2010) and to make recommendations to improve compliance.
Methods Prospective audit was conducted between January 2011 to August 2011 and 48 newborn infants with jaundice in postnatal ward were randomly selected and included. Data was collected using proforma, baby/maternal notes and blood results on computer system.

Results Poor performance was demonstrated in identifying the risk factor for jaundice-whether baby's previous sibling had jaundice requiring treatment ( $15 \%$ as compared to $100 \%$ standard). There was $90 \%$ compliance with NICE guidelines (standard 100\%) with regards to serum bilirubin (SBR) measured for infants with jaundice $<24$ hours. $83 \%$ of infants $(29 / 35)$ with jaundice onset $>24$ hours had transcutaneous bilirubinometry(TCB) measurement which reduced the number of blood tests performed on these infants. Only $75 \%$ of the parents of infants with jaundice received information leaflets on newborn jaundice.

\section{Conclusion}

1. When used appropriately, TCB reduces need for invasive blood tests and the workload of paediatric doctors on postnatal wards. The midwives and junior doctors should receive training for TCB use and management of jaundice in accordance with NICE guidelines.

2. All parents of newborn infants with jaundice should receive NICE information leaflets on jaundice to increase awareness of the condition.

\section{8 \\ ASSOCIATION BETWEEN DELAYED PASSAGE OF MECONIUM AND NEONATAL JAUNDICE IN AN UNIVERSITY HOSPITAL IN BRAZIL}

doi:10.1136/archdischild-2012-302724.1328

1.2SS Nader, 'PDJH Nader, 'DF Dolvitsch, 'H Raymundo Chinazzo. 'ULBRA, ${ }^{2}$ HU ULBRA/Mãe de Deus, Canoas, Brazil

Background and Aims Meconium contains large amounts of bilirubin and its retention leads to increased of enterohepatic circulation contributing to neonatal hyperbilirubinemia. The objective of study is investigate the relation of delayed passage of meconium and neonatal jaundice.

Methods This is a cross sectional study realized between August 2011 and March 2012, in rooming-in newborns at Universidade Luterana do Brasil Hospital. All the babies born at term, without hemolytic disease, no fetal anomalies, apgar greater than 7 in the fifth minute and breastfeeding only. The serum bilirubin levels (TBLs) were obtained by the apparatus Dräger Jaundice Meter JM-103®. The transcutaneous bilirubin variable was stratified according to the Bhutani nomogram. Were mensured TBLs between 24 and 59 hours of life. The protocol was approved by the institutional review board, and the parents' written consent was obtained.

Results Groups were similar regarding demographics and clinical characteristics. Among the 670 infants studied 118(17.9\%) have meconium amniotic fluid (MAF). The TBLs were lower in neonates with higher gestational age $(p<0.001), \operatorname{MAF}(p=0.007)$ and weight loss less than $7.5 \%$ at discharge ( $\mathrm{p}=0.001)$. The TBLs, in neonates at $\geq 37$ hours of life, were lower in the MAF group when compared with the group not MAF ( $p=0.023$ ). There is no significant association with the classification by Bhutani nomogram and the number of evacuations at discharge $(\mathrm{p}=0,051)$.

Conclusions The presence of MAF showed lower TBLs. Babies with weight loss $\geq 7.5 \%$ have higher probability to have greater TBLs. Futher studies are needed for definite results.

\section{COMPARISON OF TRANSCUTANEOUS BILIRUBINOMETER WITH SERUM BILIRUBIN IN NEONATES}

doi:10.1136/archdischild-2012-302724.1329

BR Oandalji. Peadiatrics, Ministry of Health, Amman, Jordan 
Background and Aim Serum bilirubin measurement is still the cornerstone method to assess hyperbilirubinemia in NN this method is invasive, painful, costly, needs more than one prick and may need to anemia due to repeated blood sampling.

Aim To compare trancutaneous bilirubin measurment with serum bilirubin.

Patients and Methods An average of three readings at different sites on the skin of NN using the bilirubinometer was taken babies included were FT, with indirect hyperbilirubinaemia, not exposed to phototherapy. Blood samples were collected at the same time for comparison.

Results A total of 101 measurements for each method were performed 67 babies were $>2.5 \mathrm{~kg}, 34$ babies were $<2.5 \mathrm{~kg}$. Age range was few hrs to 14 days.

There was excellent matching between the two methods of bilirubin measurement regardless of gestational age, birth weight, sex, post natal age.

Conclusion Transcutaneous bilirubin measurement is an alternative reliable way to assess hyperbilirubinaemia that avoids the drawbacks of blood sampling It is highly recommended in OPD, ER, NNU.

\section{ASSOCIATION OF TRANSCUTANEOUS CARBON MONOXIDE AND BILIRUBIN LEVELS IN HEALTHY TERM NEWBORNS}

doi:10.1136/archdischild-2012-302724.1330

D Gonulal, B Bilgin, 0 Altun Koroglu, M Yalaz, N Kultursay. Neonatology, Ege University Faculty of Medicine, Izmir, Turkey

Background and Aims End tidal measurement of carbonmonoxide (CO) as a side product of hemoglobin turn over may be an indicator of bilirubin production and hemolysis. We aimed to evaluate the association of transcutaneus $\mathrm{CO}$ measurement and bilirubin levels in healthy term newborns as an alternative to end tidal CO.

Methods A total number of 390 infants were recruited in the study. Infants who were born in our hospital $(n=340)$ were followed by daily transcutaneus $\mathrm{CO}$ and capillary bilirubin measurements starting from birth to discharge. Remaning 50 infants were admitted for hyperbilirubinemia treatment after 72 hours of life. Transcutaneous CO measurements were done by Masimo pulse oxymeter. Results Capillary bilirubin and transcutaneus CO levels on 24 and 48 hours of life were positively correlated (Spearman correlation coefficients $r=0.21$ and 0.87 respectively, $p$ values for both analyses $<0.001)$. Bilirubin levels and transcutaneous CO measurements were levels were also positively correlated in infants admitted for hyperbilirubinemia (Spearman correlation coefficient $r=0.41$, $p<0.001$ ).

Conclusion Our data showed positive and statistically significant correlations between bilirubin and transcutaneous $\mathrm{CO}$ measurements not in only healthy term newborns but also in newborns with hyperbilirubinemia. Therefore transcutaneous $\mathrm{CO}$ measurement deserves attention in order to obtain threshold levels for prediction of severe hyperbilirubinemia.

\section{PERCUTANEOUS TRANSHEPATIC ULTRASOUND-GUIDED CARDIAC CATHETERIZATION IN A FETAL LAMB MODEL}

doi:10.1136/archdischild-2012-302724.1331

${ }^{1,2} \mathrm{~A}$ Edwards, ${ }^{3} \mathrm{~S}$ Menahem, ${ }^{1}$ I Nitsos, ${ }^{4} Y$ Chan, ${ }^{1} \mathrm{~A}$ Veldman, ${ }^{5} \mathrm{D}$ Schranz, ${ }^{1} \mathrm{~F}$ Wong. ${ }^{1}$ The Ritchie Centre, Monash University; ${ }^{2}$ Perinatal Services, ${ }^{3}$ Fetal Cardiac Unit; ${ }^{4}$ Department of Pathology, Monash Medical Centre, Melbourne, VIC, Australia; ${ }^{5}$ Department of Paediatric Cardiology, Justus Liebig University, Giessen, Germany

Background Fetal cardiac intervention may alter the progression of cardiac conditions. Fetal heart access by direct puncture of the fetal heart under ultrasound guidance has been established, but with considerable technical difficulties. We aim to investigate the feasibility of fetal cardiac access using a percutaneous transhepatic approach in the mid-gestational fetal lamb, as a model for human fetus.

Method Eight fetal lambs of 95-97 days gestation (term 147 days) were studied. Under ultrasound guidance, the fetal right hepatic vein was punctured percutaneously, using a 16GA intravenous cannula (BD Angiocath). A coronary catheter (FineCross ${ }^{\mathrm{TM}} \mathrm{MG}$, Terumo) was inserted into the 16 GA cannula, over a 0.014inch guidewire, and advanced into the inferior vena cava and the right atrium. Contrast was injected to document position of the catheter. Three fetal lambs were to be euthanized at the end of the procedure to evaluate blood loss. The rest were to deliver vaginally at term, and euthanized for postmortem examination.

Results Percutaneous fetal cardiac access was successful in 7 out of the 8 fetuses. All 4 heart chambers were catheterized in the last 3 fetuses. One fetus died during the procedure, post-mortem showed pericardial and peritoneal haemorrhage. The other two fetuses dedicated for immediate post-mortem, after having survived the procedure, had small haemoperitoneum. Averaged fetal weight was $1027 \pm 153$ g. All other lambs were born normally at term.

Conclusion Ultrasound-guided fetal cardiac catheterization through a percutaneous transhepatic approach is feasible. Our experience provides a potentially safer route for human fetal cardiac intervention at midgestational age

\section{PREVALENCE OF ENTERIC COLONIZATION WITH VANCOMYCIN-RESISTANT ENTEROCOCCI IN INTENSIVE CARE UNITS IN SHIRAZ, IRAN}

doi:10.1136/archdischild-2012-302724.1332

${ }^{1} A$ Afkhamzadeh, ${ }^{2} \mathrm{~A}$ Delpisheh. ${ }^{1} K u r d i s t a n$ University of Medical Sciences, Sanandaj; 2llam University of Medical Sciences, Ilam, Iran

Background and Aims Vancomycin-resistant enterococci (VRE) are among the most feared hospital pathogens. The present nested case-control study aimed to determine the prevalence and risk factors of enteric colonization of VRE in ICUs in a tertiary hospital in Iran

Methods Serial rectal swabs were obtained every five days from all hospitalized patients (70 cases) in six ICUs and one CCU until patient's discharge. During the study 100 samples of rectal swab in sterile situation were taken. Then enterococci detection and susceptibility were done by disk diffusion and MIC dilution method.

Results A total of 9 of the 70 patients (12.85\%) were colonized with VRE. five patients who had at least a negative test for VRE - at the time of hospitalization- were colonized with resistant enterococci which demonstrated an incident rate of colonization as $7 \%$ at three months of study period. Univariate analysis showed that prior antibiotic consumption, vancomycin and third generation cephalosporins, and duration of vancomycin use were significant risk factors for VRE colonization $(p<0.05)$. There were no significant association between prevalence of VRE and variables such as age, ward, history of admission, history of antibiotic use, duration of hospitalization and underlying disease $(p>0.05)$. The logistic regression showed that the consumption of vancomycin was the independent risk factors for VRE colonization

Conclusions Prevalence of VRE in ICUs in the present study was high in consistence with national studies confirming that this organism might be endemic in tertiary large hospitals in Iran. INFECTIONS IN NEWBORN INFANTS ADMITTED TO NEONATAL INTENSIVE CARE UNIT

doi:10.1136/archdischild-2012-302724.1333 